\title{
Gastric Yolk Sac Tumor: A Case Report and Review of the Literature
}

\author{
Yeon Soo Kim', Seok Hyun Kim², Jae Koo Seong², Byung Seok Lee², Hyun Yong Jeong ${ }^{2}$ and Kyu Sang Song ${ }^{3}$
}

Department of 'Internal Medicine, Daejeon St. Mary's Hospital, College of Medicine, The Catholic University of Korea, Daejeon, Korea; Departments of 'Internal Medicine and 'Pathology, Chungnam National University College of Medicine, Daejeon, Korea

Gastric yolk sac tumors are extremely rare and their prognosis is poor; most patients have widespread metastases at the time of diagnosis. The treatment of gastric yolk sac tumors consists of aggressive chemotherapy combined with radiotherapy and surgery. Here, we first report a case of gastric yolk sac tumor presenting as an early gastric cancer that was cured after a gastrectomy with lymphadenectomy. (Korean $\mathbf{J}$ Intern Med 2009;24:143-146)

Keywords: Endodermal sinus tumor; Stomach neoplasms; alpha-Fetoproteins

\section{INTRODUCTION}

Germ cell tumors predominantly arise in the testes or ovaries, and they also occur in extragonadal regions, including the mediastinum, the retroperitoneum, the sacrococcygeal region and the intracranial region. Gastric germ cell tumors, and especially yolk sac tumors, are very rare and only few cases have been reported in the English literature [1-7]; most of these patients have widespread metastases at the time of diagnosis, so they have poor survival. The treatment of gastric yolk sac tumor is aggressive chemotherapy combined with radiotherapy and surgery. We report here on a 61-year-old man with a yolk sac tumor that presented as early gastric cancer. This is the first report of a case of gastric yolk sac tumor presenting as a localized polypoid mass, and this patient was cured after performing gastrectomy with lymphadenectomy.

\section{CASE REPORT}

A 61-year-old man was transferred to the Department of Internal Medicine under the presumptive diagnosis of early gastric cancer, which was found accidentally during a routine medical exam. Endoscopic examination showed a round polypoid mass with a reddish surface and a central depression $(1.0 \times 0.8 \mathrm{~cm})$ at the posterior wall of the greater curvature and at the mid body (Fig. 1A). A computed tomographic scan of the abdomen and pelvis showed a polypoid mass at the greater curvature of the mid body, but any lymph node enlargement was not found (Fig. 1B). The level of serum alpha-fetoprotein (AFP) was elevated to $50 \mathrm{ng} / \mathrm{mL}$, whereas the serum CEA and human chorionic gonadotropin levels were within the normal limits. Under the diagnosis of early gastric cancer, we performed endoscopic mucosal resection (EMR: strip biopsy method). During resection, mass base was found to have fibrotic changes, so we performed piecemeal dissection of the tumor in three pieces. The post-EMR pathologic findings confirmed a yolk sac tumor with invasion to the submucosa and the resection margin was positive. The 

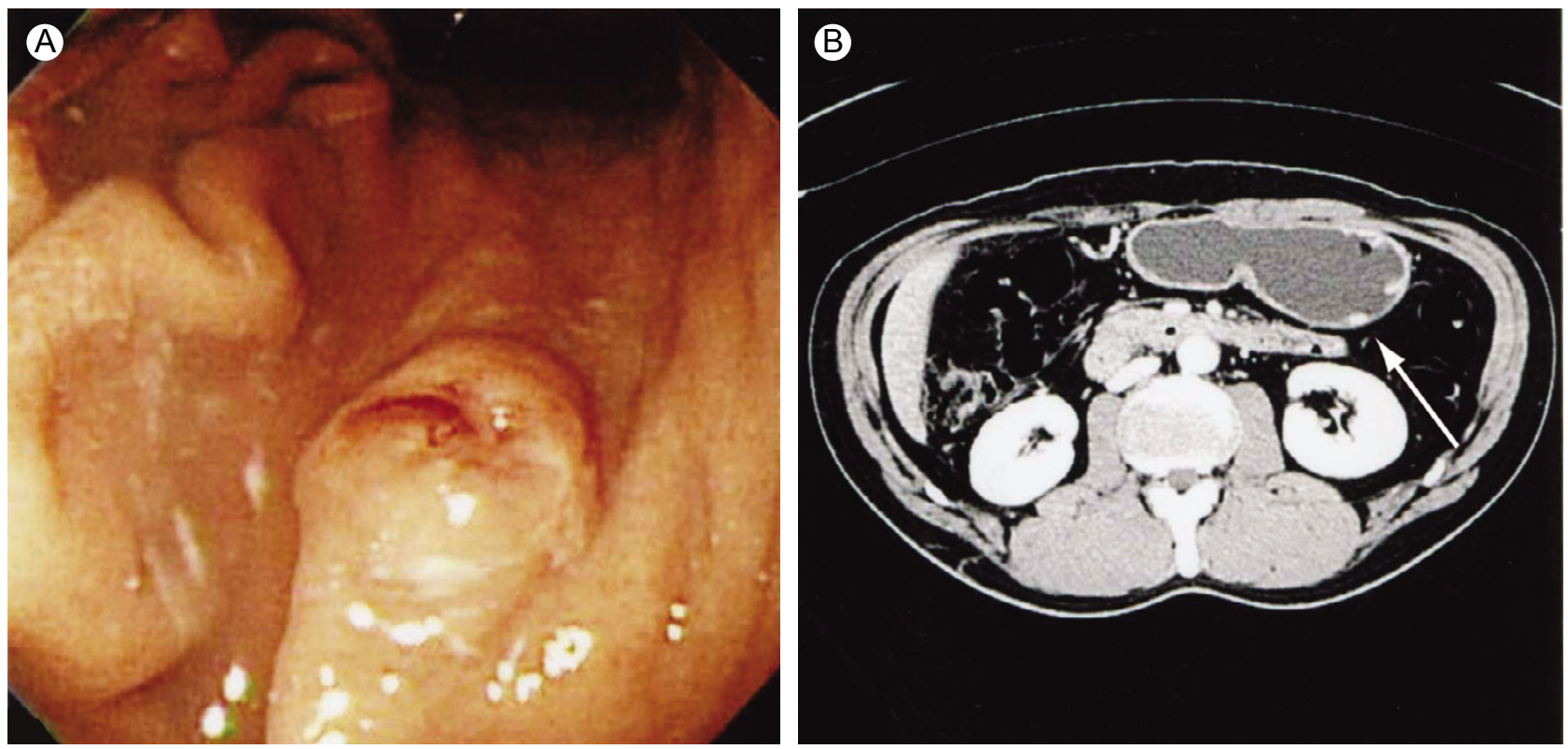

Figure 1. (A) The endoscopic findings before treatment show a $1 \times 0.8 \mathrm{~cm}$ sized polyp-like mass at the greater curvature of the mid body. (B) Abdomen-pelvic computerized tomography displays a small polyp-like elevation with enhancement at the greater curvature of the stomach.
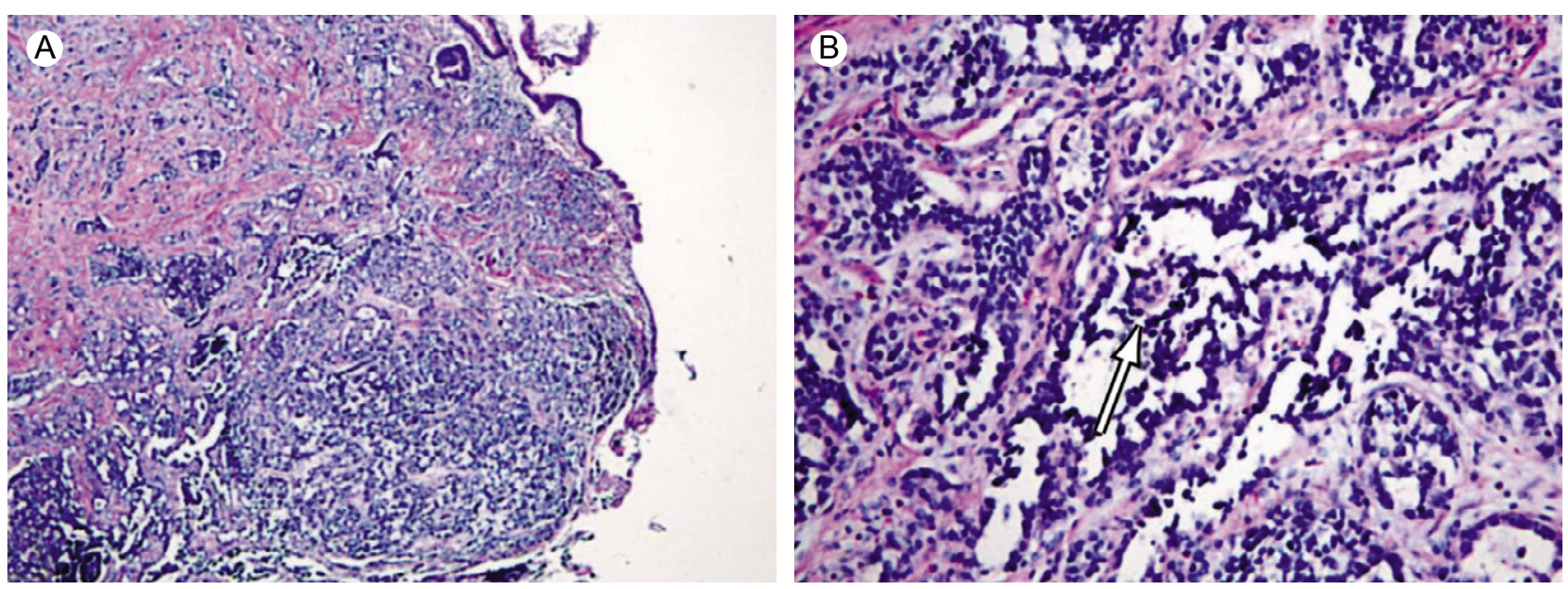

Figure 2. (A) Mucosectomy specimens show a lace-like (reticular) network of medium-sized cuboidal or flattened malignant cells and papillary structures ( $\times 40, \mathrm{H} \& \mathrm{E}$ stain). (B) Some of the malignant cells display Shiller-Duval bodies (×400, arrow).

mucosectomy biopsy of the stomach consisted of three fragments and the largest measured $1.2 \times 1 \mathrm{~cm}$ in size. A majority of the tumor showed a lace-like (reticular) network of medium-sized cuboidal or flattened malignant cells (Fig. 2A) and also papillary structures with formation of Shiller-Duval bodies (Fig. 2B). Other components of germ cell tumors, such as embryonal carcinomas and choriocarcinomas, were not identified. On the immunohistochemical staining, pancytokeratin was diffusely positive, and AFP and A1-antitrypsin were focal positive (Fig. 3). Three weeks later, we performed segmental gastrectomy with lymph node resection. However, the biopsy after surgery showed no residual tumor and no tumor in the lymph nodes. After the operation, the serum
AFP level was decreased to the normal value. The patient's general condition became very good 3 months later after surgery. Tumor recurrence was not found on the followup endoscopy and computed tomographic scan.

\section{DISCUSSION}

Primary germ cell tumors, and especially yolk sac tumors, are very rare forms of stomach malignancy and only few cases have been reported in the English literature [1-7]. The origin of a gastric yolk sac tumor has not yet been determined. The germ cells first appear from the primitive streak, the splanchnopleure and the allantoic 

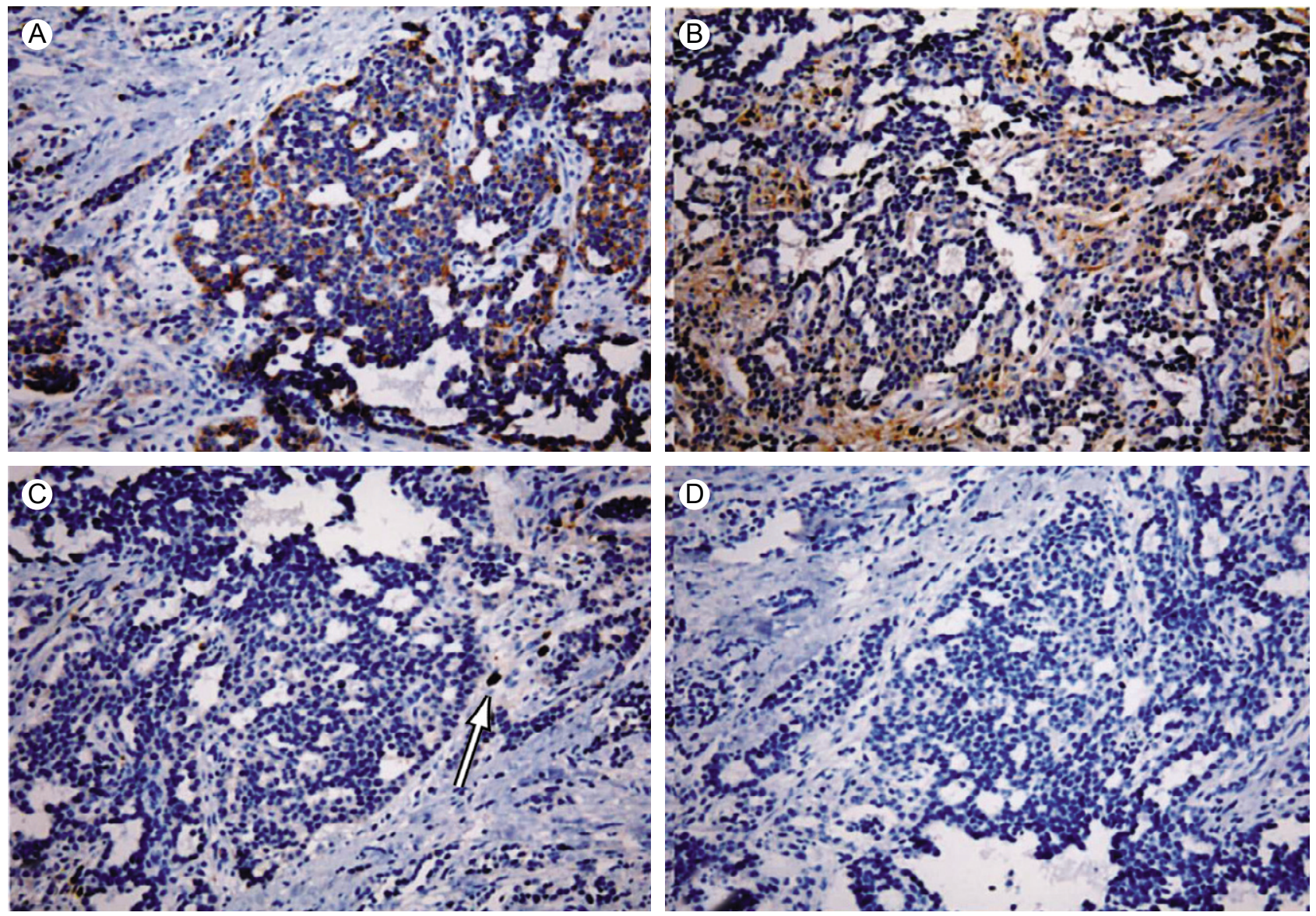

Figure 3. Immunohistochemical staining ( $\times 100)$. (A) Pancytokeratin shows diffusely positive staining. (B) AFP shows focal positive staining. (C) A1-antitrypsin shows focal positive staining. (D) CEA shows negative staining.

membrane of the embryo, and they migrate toward the gonadal ridge, but some of these germ cells may remain at ectopic sites. Yolk sac tumor of the stomach may arise from migrated germ cells, which is similar to other extragonal germ cell tumors [8]. However, some reports have suggested that the stomach arises from the foregut with all of the cells containing the complete genetic material, and the tumor cells may dedifferentiate to form the yolk sac phenotype [1].

Most of the previous reports of yolk sac tumor were the cases that presented with both malignant germ cell tumor and adenocarcinoma, and only 2 cases appeared to be a pure yolk sac tumor, without adenocarcinoma [3,7]. The prognosis of gastric yolk sac tumor is very poor because many of these patients have widespread metastases at the time of diagnosis; fortunately in our case, the tumor presented as a small polypoid mass that simulated early gastric cancer without lymph node or distant metastasis. We expected that our case would have a good prognosis since the tumor was completely resected at a rather early stage.
Histologically, yolk sac tumor has the features of an endodermal sinus tumor, including a reticular pattern (a clear-cell endoblastic pattern) with glomerulus-like structures (Schiller-Duval bodies) [1]. Also, yolk sac tumor cells produce immunoreactive AFP, so the serum AFP levels are commonly high, and AFP can be demonstrated immunohistochemically within the tumor [4]. Our case was pure yolk sac tumor that was composed of a reticular network of cuboidal malignant germ cells with typical Schiller-Duval bodies, and some of which were focal positive on immunohistochemical staining for AFP and alpha1-antitrypsin. The patient had increased serum AFP before surgery and this decreased to nearly the normal range after surgery.

We report here, for the first time in Korea, on a case of gastric yolk sac tumor presenting as early gastric cancer and the patient was cured after gastrectomy with lymphadenectomy. In contrast to the cases previously reported in literature, our case seems to have a good prognosis because of the early diagnosis and curative resection. The serum levels of AFP could be a useful 
marker of the clinical course for patients suffering with gastric yolk sac tumor.

\section{REFERENCES}

1. Garcia RL, Ghali VS. Gastric choriocarcinoma and yolk sac tumor in a man: observations about its possible origin. Hum Pathol 1985;16:955-958.

2. Motoyama T, Saito K, Iwafuchi M, Watanabe H. Endodermal sinus tumor of the stomach. Acta Pathol Jpn 1985;35:497-505.

3. Zamecnik M, Patrikova J, Comolcak P. Yolk sac carcinoma of the stomach with gastrin positivity. Hum Pathol 1993;24:927-928.

4. Wang L, Tabbarah HJ, Gulati P, Rice S, French SW. Gastric adenocarcinoma with a yolk sac component:a case report and review of literature. J Clin Gastroenterol 2000;31:85-88 .

5. Suzuki T, Kimura N, Shizawa S, et al. Yolk sac tumor of the stomach with adenocarcinomatous components: a case report with immunohistochemical analysis. Pathol Int 1999;49:557-562.

6. Puglisi F, Damante G, Pizzolitto S, et al. Combined yolk sac tumor and adenocarcinoma in a gastric stump: molecular evidence of clonality. Cancer 1999;85:1910-1916.

7. Kanai M, Torii A, Hamada A, et al. Pure gastric yolk sac tumor that was diagnosed after curative resection. Int JGastrointest Cancer 2005;35:77-81.

8. Robbins SL, Contran RS. Robbins pathologic basis of disease. 7 th ed. Philadelphia:W.B. Saunders, 2004. 